\title{
Further Experimental Studies on the Production of Pulmonary Infarction
}

\section{Especially from Coagulative and Fibrinolytic Studies}

\author{
Hiroshi Hasegawa, M.D., Naoyoshi Watanabe, M.D., Nobuo \\ Watanabe, M.D., Hironobu Kakizaki, M.D., Hajime \\ Murata, M.D., and Makoto Murao, M.D.
}

\section{SUMMARY}

Attempts were made to clarify the mechanism with which the pulmonary infarction occurs, from coagulofibrinolytic point of view.

The following results were obtained:

(1) Pulmonary infarction could be produced at such a high rate as $74.4 \%$ through infusion of blood clots 2 days after intravenous injection of Lycopodium spores, when the coagulation activity was on the highest grade and the fibrinolytic activity was on the lowest grade.

(2) Severe hemorrhage was observed in the post-mortem examination of the lungs 24 to 48 hours after infusion of the blood clots. The embolus remained thereafter, but the hemorrhage tended to diminish.

(3) Consumption of coagulative factors and fibrinolytic factors was observed in the pulmonary venous blood than in the pulmonary arterial blood 3 hours after infusion of blood clots. Consumption of the coagulative and fibrinolytic factors and increase of F.D.P. were observed 12 to 24 hours after the infusion, although there were more or less differences depending upon experimental methods.

(4) Consumption of free-type activator, increase of bond-type activator, and formation of marked focal lysis ring examined by means of fibrinolysis autograph were observed in tissue samples examined 24 to 48 hours after the infusion of blood clots.

(5) It was assumed that a close relationship exists between the production of pulmonary infarction and the disorders of the coagulationfibrinolytic system, especially the fibrinolytic factors including those in the blood and tissue samples, mainly examined around 24 hours after infusion of blood clots.

\section{Additional Indexing Words :}

Lycopodium spores P.T.T. Fibrinogen Total plasma plasmin activity Platelet adhesiveness Platelet aggregation Fibrinolysis autograph F.D.P.

From the First Department of Medicine, Hokkaido University School of Medicine, Sapporo, Hokkaido.

Received for publication January 5, 1971. 
UR studies are in progress to evaluate on the relationship between the experimental production of pulmonary infarction and the disorder in the coagulation-fibrinolytic system. Wc have already reported on our findings that the intravenous injection of blood clots alone into normal rabbits' ear vein did not produce any pulmonary infarction, and that the pulmonary infarction could be produced to such a high extent as $62.5 \%$ by infusion of blood clots into jugular vein with an anti-plasmin agent following 5 successive days intravenous preinjection of Lycopodium spores. The high rate was even so higher as to reach $88.9 \%$ when the combination of blood clots and antiplasmin was additionally coupled with intravenous administration of human serum.1) In this connection, we have also pointed out that the factors for production of pulmonary infarction are not only circulatory disturbances or damages in the vascular beds, but also the hypercoagulative and hypofibrinolytic activity prior to the infusion of blood clots, or the activation of tissue activator following these conditions. ${ }^{13}$ A series of these experiments was conducted 5 days after the intravenous injection of Lycopodium spores, but we found that the mortality of the experimental animals was higher on the 2nd day after its intravenous injection when the blood coagulation was on the highest grade and the fibrinolytic activities were on the lowest grade, if the blood clots was infused on that day. In this experimental series, we have examined the effects of blood clots infused on the 2nd day after Lycopodium spores injection on the occurrence rate of pulmonary infarction, and changes in the coagulation-fibrinolytic system, and others.

\section{Materials and Methods}

1) Experimental animals :

Rabbits with body weight of $2.2 \mathrm{Kg}$. to $2.5 \mathrm{Kg}$. were employed.

2) Blood collection method:

Blood samples were collected from ear vein (for successive values before and after the procedure) and outer jugular vein (for values before infusion of blood clots) and through puncture of right and left ventricle of the heart (when thorax was opened under controlled respiration).

3) Examinations on the coagulation-fibrinolysis:

a) P.T.T. :

Fifty times diluted Tachostiptan (a product of Sumitomo Chemical) was used for this test.

b) Prothrombin time:

Lyoplastin (a product of Mochida Pharm. Co.) was employed for this test.

c) T.G.T. :

In accordance with Hicks-Pitney method, ${ }^{21}$ the 3-min. values were examined for comparison.

d) Plasma fibrinogen : 
This was determined by Tyrosine method. ${ }^{3)}$

e) Total plasma plasmin activity:

This was determincd by the standard plate method using bovine fibrinogen.

f) Number of platelets :

This was counted by the Brecher-Cronkite method. ${ }^{4)}$

g) Platelet aggregation :

EEL aggregation meter, model 169 was employed for this test.

h) Platelet adhesiveness :

Glass filter 3G-I was employed for this test.

i) Determination method of tissue activator:

(i) Extraction method:

The thorax of test animals was opened under respiration control using a respirator for small experimental animals. The lungs were perfused with $250 \mathrm{ml}$. of physiological saline through pulmonary arteries. Thence, the lungs were picked up and extracted individually using $2 \mathrm{M}$. urea and $1 \mathrm{M}$. KSCN with 80 times diluted volume for wet-weight of the tissue. The extract was then centrifuged and its supernatant was allowed to drop upon the standard plate, whereupon the lysis area was determined.

(ii) Fibrinolysis autograph:

Immediately following extraction, the lungs were subjected to rapid refrigeration, followed by preparation of tissue slices using the cryostat. This was placed on a film of fibrin prepared in advance and allowed to 6 subsequent hours incubation. The product was then fixed with formalin vapor and stained with hematoxylin and eosin for observation of focal lysis ring. The fibrinogen used was the product or rabbit origin prepared by Mann Research Laboratories.

j) F.D.P. determination :

This was determined by means of the capillary method in accordance with the tanned red cell hemagglutination inhibition method. The minimum detectable concentration was $10 \mu \mathrm{g} . / \mathrm{ml}$. of F.D.P.

4) Method for infusion of Lycopodium spores:

Lycopodium spores were infused into ear vein in a dose of $6 \mathrm{ml}$. suspension of physiological saline containing $10 \mathrm{mg} . / \mathrm{ml}$. thereof.

5) Method of infusion of blood clots:

Two lumps of human white thrombus each weighing approximately $30 \mathrm{mg}$. were prepared, and infused into outer jugular vein.

6) Method for preparation of pulmonary infarction :

In accordance with the method described in 4), blood clots were infused on the 2nd day of intravenous injection of Lycopodium spores. Postmortem examinations of the experimental animals were conducted at some hours' or days' interval. The blood samples collected in accordance with the method 2) were employed for determination of coagulation-fibrinolytic activities. The tissue fibrinolytic activities were determined in accordance with the method quoted in the paragraphs 3)-(i), -(ii) and -(iii).

\section{RESULTS}

1) Changes in the blood coagulation as well as fibrinolytic activities upon production of microembolism following intravenous injection of Lyco- 

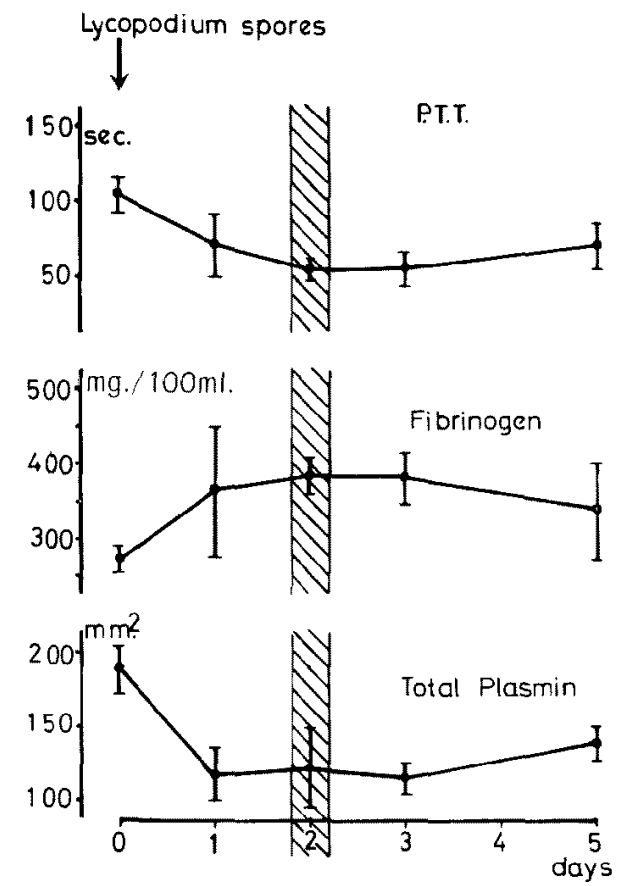

Fig. 1. Changes in the levels of P.T.T, fibrinogen and total plasmin following intravenous injection of $6 \mathrm{~mL}$. of Lycopodium spores $(10 \mathrm{mg} . / \mathrm{ml}$.).

podium spores:

In the results obtained up to the 5 th day, P.T.T. shortening and fibrinogen increase were maximized on the 2 nd day, while the drop in the total plasmin activity was maximized on the 3rd day, and all of the maximum counts were found statistically significant (Fig. 1).

2) Changes of the coagulative and fibrinolytic activities and pulmonary infarction following infusion of blood clots 2 days after intravenous injection of Lycopodium spores (using 47 rabbits):

a) Rate of production of pulmonary infarction :

Mortality following anesthesis and/or infusion of blood clots was 4 (8.6\%) of all rabbits examined, while hemorrhagic infarction was produced in 32 out of 43 rabbits $(74.4 \%$ ), involving 11 in their right lungs, 6 in their left lungs, and 15 in their both lungs. The infarction was observed in 30 out of 36 which were subjected to post-mortem examination by the 7 th day $(83.3 \%)$. The hemorrhage was slight up to 3 hours after infusion of blood clots, but the most notable 24 to 48 hours after the infusion. The extent of hemorrhage became slight after the 4th day. The embolus existed in most of the 7 rabbits which were subjected to post-mortem examination after the 8th day, while the infarction was observed in only 2 rabbits $(28.5 \%)$, and they were not neces- 
Table I. Post-mortem Findings in the Lungs and Frequency of Onset of Pulmonary Infarction after Infusion of Blood Clots

\begin{tabular}{|c|c|c|c|c|c|c|c|c|c|c|c|c|c|}
\hline \multirow{2}{*}{ Infarction } & \multicolumn{11}{|c|}{ Day } & \multirow{2}{*}{$\begin{array}{l}\text { Total } \\
\text { No. }\end{array}$} & \multirow{2}{*}{$\%$} \\
\hline & $<1$ & 1 & 2 & 3 & 4 & 5 & 6 & 7 & 8 & 9 & $10<$ & & \\
\hline Right L. & & & 1 & 1 & 1 & 3 & 1 & 4 & & & & 11 & 25.6 \\
\hline Left $L$. & & & 1 & 1 & 2 & & & 2 & & & & 6 & 14.0 \\
\hline Both L. & 3 & 4 & 1 & 1 & & 1 & 1 & 1 & 2 & & & 15 & 34.8 \\
\hline None & & 1 & 1 & & 1 & & 1 & 2 & & 2 & 3 & 11 & 25.6 \\
\hline Total No. & 3 & 5 & 4 & 3 & 4 & 4 & 3 & 10 & 2 & 2 & 3 & 43 & 100.0 \\
\hline
\end{tabular}

sarily clear to be detected (Table I).

b) Changes in the coagulative and fibrinolytic system:

(i) P.T.T.

It showed a tendency to shorten on 3 hours after the infusion, but elongate afterwards on the first day. No statistic difference was observed in P.T.T. valucs of the pulmonary arterial blood with those of the venous one except those on the 4th day. The P.T.T. showed a more prolongation in the pulmonary venous blood than in the pulmonary arterial blood after 3 hours following the infusion. After one day's lapse from the infusion, the P.T.T. values were more prolonged in the pulmonary arterial blood than in the venous blood, and this trend remained thereafter.

ii) T.G.T.

No statistically significant difference could be observed between the pulmonary arterial blood and venous blood. After 3 hours' lapse, however, a marked trend of larger drop in the T.G.T. activity was observed in the pulmonary venous blood than in the arterial one (Fig. 2).

iii) Prothrombin time

No statistically significant difference could be observed between the pulmonary arterial blood and venous blood. After 3 hours' lapse, however, a marked trend of larger drop in the prothrombin time was observed in the pulmonary venous blood than in the arterial one, and this remained until the 2nd day following the infusion.

\section{iv) Fibrinogen}

The fibrinogen was decreased more in the pulmonary venous blood 3 hours after the infusion than in the pulmonary arterial blood, and statistically significant difference could be observed between the arterial blood and venous blood. Thereafter both arterial and venous blood showed a trend of decrease until the 2nd or the 3rd day following the infusion (Fig. 2). 

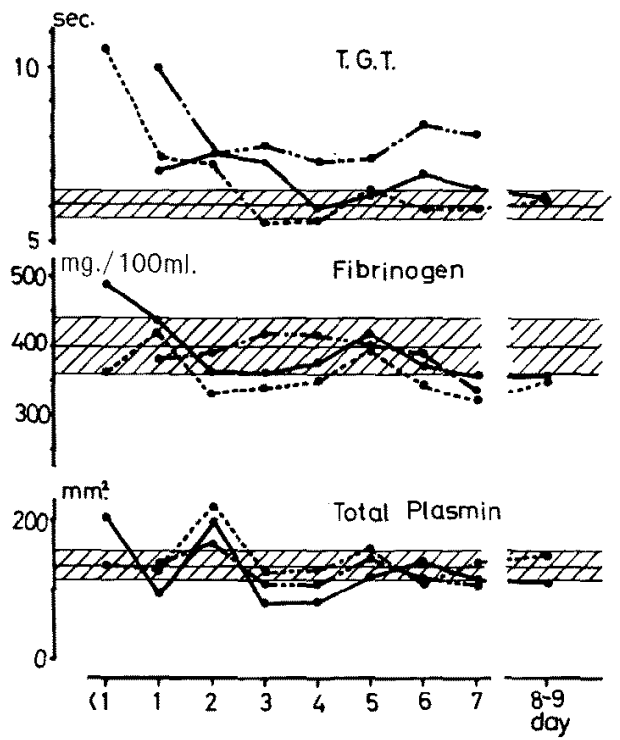

Fig. 2. Changes in the levels of T.G.T., fibrinogen and total plasmin following infusion of blood clots.

P.A. Blood

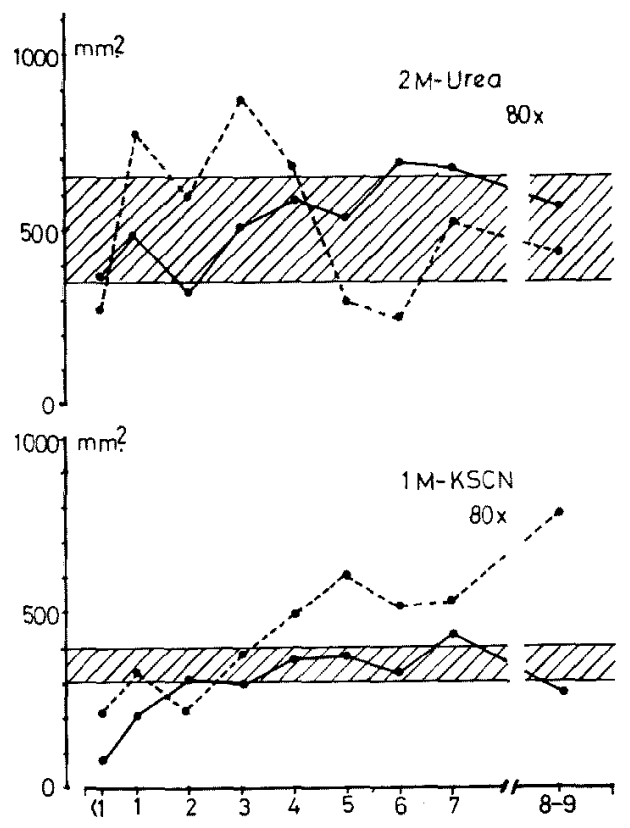

Fig. 3. Changes in the levels of tissue activators following infusion of blood clots.

Infarction Area ......; Other Area - - $\bullet-$ 


\section{v) Total plasmin activity}

No statistically significant difference could be observed between the arterial and the venous blood. After 3 hours' lapse, however, a marked trend of larger drop in the total plasmin activity was observed in the pulmonary arterial blood than in the pulmonary venous one. After 24 hours' lapse, this difference became reverse. This activity showed increase with 2 peaks after 3 hours' and 2 days' lapse following the infusion, but a drop in the blood sample after 24 hours' lapse (Fig. 2).

vi) Tissue activator

The tissue activator showed a higher rise of activity in the infarction area than in the non-infarction area in terms of samples extracted with $2 \mathrm{M}$. urea until 24 hours' to 4 days' lapses. This rise was higher than or at least equal to the upper limit of the normal values. Until 5 or 6 days' lapses, the activity was found to be lower than the normal value. In the sample extracted with $1 \mathrm{M}$. KSCN, the tissue activator activity was found a more increase in the infarction area in the 24 hours sample than in the non-infarction area, but this ratio became reverse after 24 hours' lapse. These activities were, however, found lower than or at least equal to the lower limit of the normal value. After 4 days lapse activities in the infarction area were found to be higher than the normal value (Fig. 3).

3) Studies on successive changes of various parameters using the venous blood in the ear of the same rabbit (using 6 rabbits):

Successive changes of parameters such as P.T.T., T.G.T., fibrinogen, and total plasmin of the venous blood in the ear of the same rabbit were compared with those of the above-mentioned values obtained in pulmonary arterial as well as venous blood of successively sacrificed animals. No statistically significant difference could be observed between the values of the venous blood in the ear and either one of the arterial and venous blood, except the T.G.T. value on the 7 th day. A trend was observed that values of the parameters of the venous blood in the ear were close to those obtained with pulmonary arterial blood (Fig. 2).

4) Changes in the coagulation-fibrinolytic activities in a short period after the infusion of blood clots (using 12 rabbits):

a) Platelets

Some indicated increase, while the other decrease in the number of platelets in the blood collected 3 hours after infusion, but the average indicated a trend of incrcase, and turned then into a trend in decrease arriving at the lowest level 24 hours after the infusion. However, it showed gradual increase thereafter. The platelet aggregation showed decrease of activity from 3 hours to 24 hours after the infusion, and gradually increased thereafter. The platelet 
adhesiveness showed a drop in the blood collected 6 to 24 hours after the infusion, and then a trend of gradual increase thereafter (Fig. 4).

b) Fibrinogen

Fibrinogen decreased in the blood samples collected 3 to 12 hours after the infusion, and increased slightly in the sample collected 24 hours after the infusion, but decreased again at 48 hours. Thereafter, it increased gradually (Fig. 5).
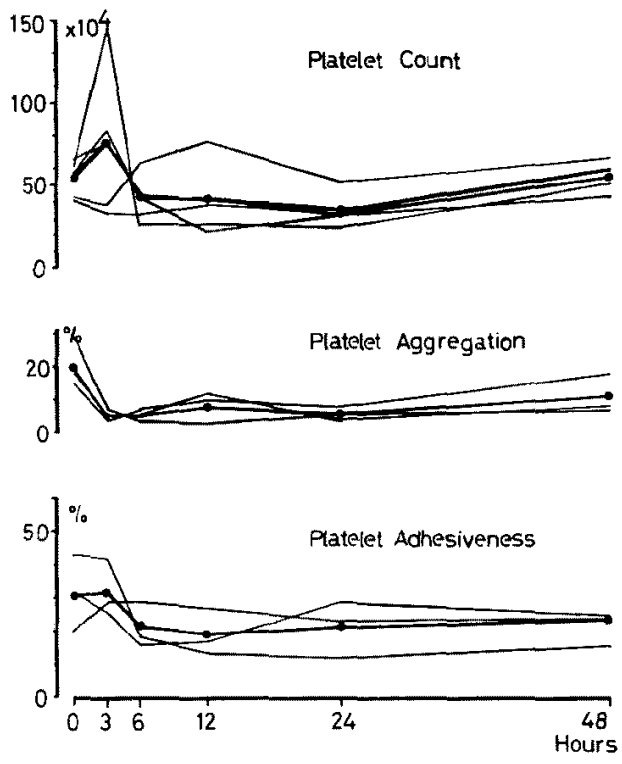

Fig. 4. Changes in the levels of platelet count, platelet aggregation and platelet adhesiveness following infusion of blood clots.

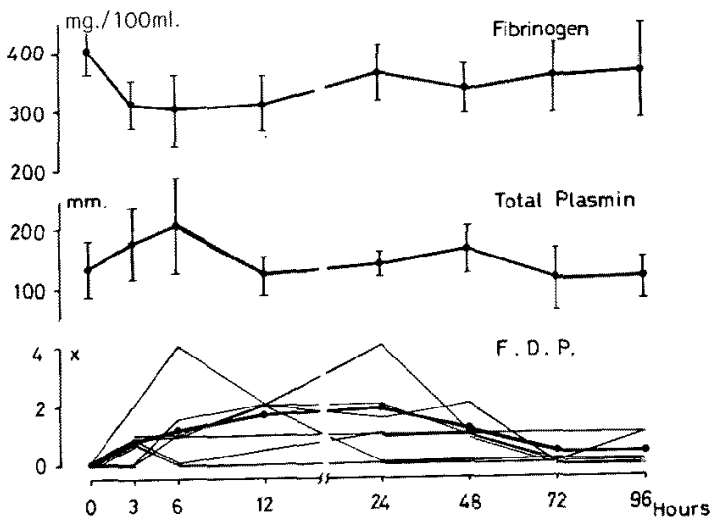

Fig. 5. Changes in the levels of fibrinogen, total plasmin and F.D.P. following infusion of blood clots. 
c) Total plasmin activity

Total plasmin activity showed the maximum value 6 and 48 hours after the infusion, but showed low levels in the samples 12 to 24 hours after the infusion, that is, a two-peaked activity curve (Fig. 5).

d) F.D.P.

F.D.P. showed a gradual increased since 3 hours after the infusion, reaching the peak at 24 hours followed by a gradual decrease thereafter (Fig. 5).

5) Fibrinolysis autograph (using 10 rabbits):

Since no focal lysis ring was observed in fibrin film when bovine fibrinogen was used, rabbit fibrinogen was employed in its stead. In the sample examined 24 hours after the infusion, focal lysis rings were observed between the embolus and the blood vessel wall, and on 48 hours after the infusion considerably many focal lysis rings were observed in the embolus and blood vessel wall and between them (Fig. 6). The focal lysis rings decreased on the 5 th and 8 th days after the infusion.

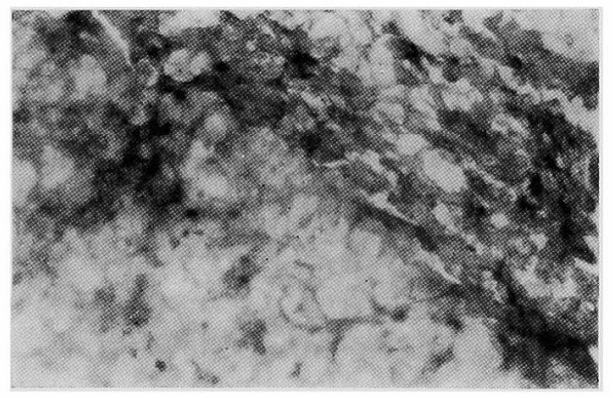

Fig. 6. Marked focal lysis rings were found in the arterial wall and embolus (6 hours incubation, $\times 200, \mathrm{H}-\mathrm{E}$ staining).

\section{Discussion}

It has already been reported that infusion of blood clots at the time of hypercoagulative and hypofibrinolytic activity 2 days after the intravenous injection of Lycopodium spores tended to cause higher mortality of rabbits. ${ }^{1)}$ However, by careful infusion of the blood clots on the 2nd day, the hemorrhagic infarction could be produced in as high a rate as $74.4 \%$. Postmortem examination of the lungs produced severer hemorrhage 24 to 48 hours after the infusion than 3 hours. The hemorrhage was gradually diminished thereafter to such an extent that, despite the existence of embolus 8 days after the infusion, any hemorrhagic patterns could hardly be detected. Thus, following infusion of blood clots, reversible pulmonary infarction could be produced, but the pulmonary infarction brought about by the administration of blood clots and t-AMCHA or serum on the 5th day following intravenous injection 
of Lycopodium spores could be considered as irreversible. From the clinical viewpoint of these facts, it may be suggested that addition of thrombus or embolus in pulmonary artery to the existing hypercoagulative and hypofibrinolytic activity is prone to produce pulmonary infarction, and tends to be a reversible infarction. It may be further suggested interestingly that, even if the extent of hypercoagulative and hypofibrinolytic activity is not so higher, the possibility of production of pulmonary infarction tends to yield the highest rate and the pulmonary infarction is prone to be irreversible, only if there are complications of circulatory disturbances or damages in the vascular beds with various cardiac and/or pulmonary diseases, blood diseases, or malignant tumors such as pulmonary carcinoma.

In view of the fact hardly significant statistic difference could be observed between the values obtained successively from venous blood of the same rabbit and those obtained with pulmonary arterial and venous bloods from each of successively sacrificed samples, studies were made on the assumptive basis that this series of results obtained from the successively sacrificed animals might be applied to the phenomena in the same individual rabbit, whereupon it was found that there were some differences between the values of pulmonary arterial blood and pulmonary venous blood in terms of P.T.T., prothrombin time, T.G.T., and fibrinogen, 3 hours after the infusion. In other words, differences in these values were observed, which were attributable to the hypercoagulation in pulmonary arterial blood and to the consumption in pulmonary venous blood, the latter having been lower activity than the former. Elongation of coagulation time or drop in the activity attributable to the consumption was observed expressed by changes in P.T.T. after lapse of 24 hours, and prothrombin time, T.G.T., and fibrinogen after lapse of 1 to 3 days following the infusion. However, despite the consumption of coagulation factors, coagulation time expressed by P.T.T. was rather shortened especially in the pulmonary venous blood from the 2 nd to the 4 th days following the infusion. This suggests that the shortening might have been due to the release of the third factor from the pulmonary infarction area. There were also difference in fibrinolytic system between the arterial and venous blood collected 3 hours after the infusion. It may be assumed that plasminogen might have been consumed in pulmonary infarction area. Total plasmin activity after 24 hours' lapse markedly decreased in both of them, particularly these activities in the pulmonary arterial blood were decreased more than those after 3 hours' lapse. This drop may be attributed to consumption of plasminogen, and the activity again increased in the blood sample collected after 48 hours' lapse. In both samples collected 24 and 48 hours' lapse, the activity was higher in the pulmonary venous blood than in the arterial one, which suggested release of tissue 
activator from the infarction area.

The tissue activator level remained relatively low in the samples extracted up to 48 hours' lapse with $1 \mathrm{M}$. KSCN. This may be attributed to consumption of free type tissue activator. The rise of activity after lapse of 5 days seemed to be attributable to recovery of tissue based on the findings upon postmortem examination of the lungs. The activity showed relatively high levels in the samples extracted from the infarction area with $2 \mathrm{M}$. urea after 1 to 4 days' lapse, which showed, however, a marked drop in the samples examined 5 or 6 days' lapse. The drop was considered to be possibly due to consumption.

Based on the above-mentioned facts, it was surmised that changes in the coagulation-fibrinolytic system during a short period after infusion of blood clots and the tissue fibrinolytic activity have much to do with production of infarction. Hence, changes in the levels of platelet, fibrinogen, total plasmin and F.D.P. during a short period of time were examined. The number of platelet increased in the sample collected 3 hours after the infusion, but decreased 6 hours thereafter due to consumption, reaching the minimum after 24 hours' lapse, followed later by gradual increase. The total plasmin activity increased after 3 hours following the infusion, and the sample collected even after 48 hours' lapse also showed an increase, which however showed a drop possibly attributable to consumption of plasminogen derived from release of activator from the infarction area 12 to 24 hours following the infusion. Fibrinogen level showed decrease after 3 hours' lapse, but also showed a temporary rise in the sample collected after 24 hours' lapse possibly due to the action of F.D.P. The level dropped again in the sample collected after 48 hours' lapse, which resumed gradual increase thereafter. The changes in the fibrinogen level might be attributed to the consumption due to secondary production of thrombus and the reproduction. The level of F.D.P. which is the degradation product of the fibrin also increased after lapse of 3 hours, reaching its maximum after 24 hours' lapse. It is considered very likely that the drop of platelet aggregation and adhesiveness must have taken place in the samples collected 3 to 6 hours after infusion due to the changes of the F.D.P. level. ${ }^{5}$ )

In view of the above-cited results of examinations of blood samples during a short period, it may be concluded that the consumption of coagulation factors including the platelets after 12 to 24 hours following infusion of blood clots, hyperfibrinolysis assumed from consumption of plasminogen, consumption of free type tissue activators (showed by the extraction with $1 \mathrm{M}$. KSGN), increase of bond-type activator (showed by the extraction with $2 \mathrm{M}$. urea), and the marked focal lysis ring certified by fibrinolysis autograph would underline the appearance of severe hemorrhagic infarction.

The mechanism of production of pulmonary infarction is characterized 
by consumption of coagulation factors due to secondary production of thrombus around the embolus on 3 hours after infusion of blood clots, and hyperfibrinolysis due to release of tissue activator from the endothelium of blood vessels around embolus, coupled with tendency for hemorrhage in the periphery from the embolus and subsequent severe consumption of coagulation factors due to release of the third factor. It is further characterized by destruction of alveolar walls and severe hemorrhage in the periphery from the embolus, owing to hyperfibrinolysis following release of tissue activators from around the embolus due to dissolution of embolus, eventually leading to production of pulmonary infarction (Fig. 7).

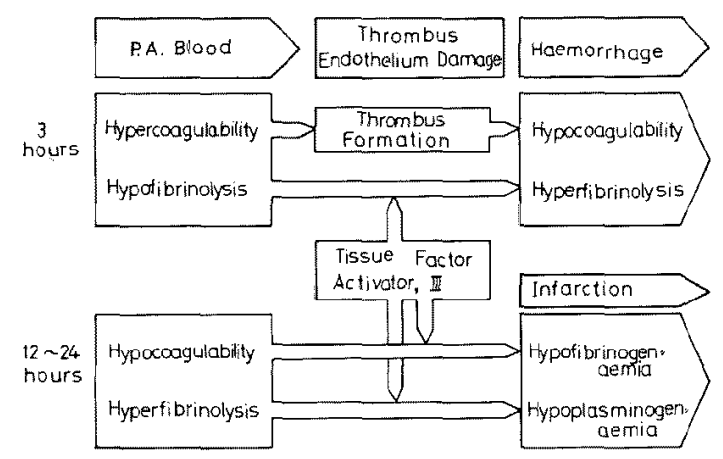

Fig. 7. Scheme of the production of pulmonary infarction.

As above mentioned, it might be justifiably claimed that the disorders of coagulation fibrinolytic system, particularly the fibrinolytic system including the blood and tissue, are closely related to the production of pulmonary infarction.

\section{REFERENGES}

1. Hasegawa, H., et al. : Jap. Heart J. 11 : 258, 1970.

2. Hicks, N. D. and Pitney, W. R.: Brit. J. Haemat. 3: 227, 1957.

3. Matsuoka, M., et al.: J. Med. Technology 2:61, 1958 (in Japanese).

4. Brecher, G. and Cronkite, E. P.: J. Appl. Physiol. 3: 365, 1950.

5. Kowalski, E. M., et al.: Thrombos. Diathes. Haemorrh. 10 : 406, 1964. 